\title{
OPEN Increasing prevalence of resistance to second-line drugs among multidrug-resistant Mycobacterium tuberculosis isolates in Kuwait
}

\begin{abstract}
Noura M. Al-Mutairi ${ }^{1}$, Suhail Ahmad ${ }^{1}$ \& Eiman Mokaddas $^{1,2}$
Molecular methods detect genetic mutations associated with drug resistance. This study detected resistance-conferring mutations in gyrA/gyrB for fluoroquinolones and rrs/eis genes for second-line injectable drugs (SLIDs) among multidrug-resistant Mycobacterium tuberculosis (MDR-TB) isolates in Kuwait. Fifty pansusceptible M. tuberculosis and 102 MDR-TB strains were tested. Phenotypic susceptibility testing was performed by MGIT 960 system using SIRE drug kit. GenoType MTBDRsl version 1 (gMTBDRslv1) and GenoType MTBDRsl version 2 (gMTBDRslv2) tests were used for mutation detection. Results were validated by PCR-sequencing of respective genes. Fingerprinting was performed by spoligotyping. No mutations were detected in pansusceptible isolates. gMTBDRslv1 detected gyrA mutations in 12 and $r$ rs mutations in 8 MDR-TB isolates. gMTBDRs/2 additionally detected gyrB mutations in 2 and eis mutation in 1 isolate. Mutations in both $g y r A / g y r B$ and $r r s / e i s$ were not detected. gMTBDRslv1 also detected ethambutol resistance-conferring embB mutations in 59 isolates. Although XDR-TB was not detected, frequency of resistance-conferring mutations for fluoroquinolones or SLIDs was significantly higher among isolates collected during 2013-2019 versus 2006-2012. Application of both tests is warranted for proper management of MDR-TB patients in Kuwait as gMTBDRslv2 detected resistance to fluoroquinolones and/or SLIDs in 3 additional isolates while gMTBDRslv1 additionally detected resistance to ethambutol in 58\% of MDR-TB isolates.
\end{abstract}

Although the incidence of active disease and deaths have slowly declined worldwide in recent years, tuberculosis (TB) is still the leading cause of death from a single infectious agent ${ }^{1}$. According to the World Health Organization (WHO), an estimated 10 million active TB disease cases (equivalent to 130 cases per 100,000 population) and $\sim 1.41$ million deaths occurred in $2019^{1}$. Furthermore, an estimated 465,000 people developed TB that was resistant to rifampin (RR-TB), and of these, nearly 363,000 (78\%) were multidrug-resistant (MDR)-TB (defined as infection with Mycobacterium tuberculosis strain resistant at least to rifampin, RIF and isoniazid, INH; the two most effective first-line drugs) cases $^{1}$. The WHO has further categorized infection with M. tuberculosis strains resistant only to RIF and INH without additional resistance to other first-line (ethambutol, EMB and pyrazinamide, PZA) drugs as uncomplicated MDR-TB. Successful treatment of uncomplicated MDR-TB is higher compared to treatment of MDR-TB resistant to additional first-line drugs ${ }^{2-5}$. It is estimated that nearly $6.2 \%$ of all MDR-TB cases have XDR-TB [MDR-TB strains additionally resistant to a fluoroquinolone (FQ) plus a second-line injectable drug (SLID); kanamycin (KAN), amikacin (AMI) or capreomycin (CAP)] and at least 1 case of XDR-TB has been reported by 131 countries/territories by the end of $2018^{6}$. Furthermore, $20.1 \%$ of 465,000 RR/MDR-TB isolates worldwide were resistant to a FQ in $2019^{1}$. Worldwide, treatment success rates for drug-susceptible TB, MDR-TB and XDR-TB have been reported as 85\%, 56\%, and 39\%, respectively ${ }^{6}$. Among MDR-TB strains, mutations in $r p o B$ mostly confer resistance to RIF while mutations in kat $G$ codon 315 (kat G), and inhA regulatory region (inhA) usually confer resistance to $\mathrm{INH}^{3}$. Early detection of $M$. tuberculosis in clinical specimens, its susceptibility to anti-TB drugs, and effective treatment are essential for global TB control efforts ${ }^{3-7}$.

${ }^{1}$ Department of Microbiology, Faculty of Medicine, Health Sciences Centre, Kuwait University, P. O. Box 24923, 13110 Safat, Kuwait. ${ }^{2}$ Kuwait National TB Control Laboratory, Shuwaikh, Kuwait. ${ }^{\boxplus}$ email: suhail.ahmad@ ku.edu.kw 


\begin{tabular}{|l|c|c|}
\hline \multirow{2}{*}{ Clinical specimens } & \multicolumn{2}{|l|}{ No. of M. tuberculosis isolates grown as } \\
\cline { 2 - 3 } & Pansusceptible strains & MDR-TB strains \\
\hline Respiratory samples & 29 & 69 \\
\hline Sputum & 5 & 9 \\
\hline Bronchoalveolar lavage & \multicolumn{2}{|l}{} \\
\hline Non-respiratory samples & \multicolumn{1}{|l}{} \\
\hline Fine needle aspirate & 7 & 11 \\
\hline Pus & 4 & 5 \\
\hline Lymph node & 1 & 3 \\
\hline Tissue biopsy & 1 & 3 \\
\hline Cerebrospinal fluid & 1 & 2 \\
\hline Pleural fluid & 2 & 0 \\
\hline Total & 50 & 102 \\
\hline
\end{tabular}

Table 1. Clinical source of pansusceptible M. tuberculosis and MDR-TB strains.

The FQs, and SLIDs (KAN, AMI or CAP) are the backbone of most treatment regimens for MDR-TB as resistance to both these drugs in MDR-TB strains defines XDR-TB ${ }^{2-4,8}$. Patients infected with M. tuberculosis isolates carrying specific gyrA (A90V, D94G, D94N and D94Y) mutations conferring high-level resistance to FQs or $\operatorname{rrs}$ (A1401G) mutation conferring high-level resistance to KAN have higher mortality rates ${ }^{9,10}$. New generation FQs (Levofloxacin, LFX, moxifloxacin, MFX and gatifloxacin, GFX) have both bactericidal and sterilizing activity against $M$. tuberculosis as they penetrate into cavitary lesions and are active against resident bacterial populations ${ }^{11}$. With the availability of two new anti-TB drugs, bedaquiline and delamanid, FQs have recently been placed ahead of SLIDs since their use is associated with a favorable outcome for MDR-TB treatment ${ }^{12}$.

Phenotypic drug susceptibility testing (DST) by automated methods such as mycobacteria growth indicator tube (MGIT) 960 system, considered reliable to determine susceptibility or resistance of $M$. tuberculosis to firstline anti-TB drugs until recently, has yielded discrepant results in recent studies ${ }^{3,4,7,8,10,13,14}$. Furthermore, these

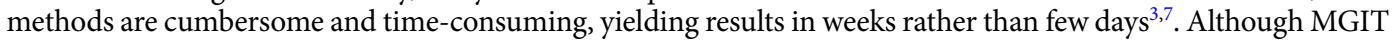
960 system has also been evaluated for FQs and SLIDs, these studies have mainly used M. tuberculosis strains exhibiting high-level resistance to these drugs ${ }^{3,7,8}$. Extensive data on rifampin-resistant ${ }^{15-19}$ and ethambutolresistant $^{20-24}$ strains indicate that $M$. tuberculosis isolates with low-level resistance to FQs/SLIDs may also yield discordant results by MGIT 960 system. Indeed, recent studies have shown that M. tuberculosis isolates with minimal inhibitory concentration (MIC) values close to the critical drug concentration (CC) and carrying specific mutations in resistance conferring ( $g y r A$ or $g y r B)$ genes yield discordant results by phenotypic methods ${ }^{25-27}$. Since treatment failure or disease relapse is nearly same for RIF-resistant isolates with 'disputed' (MICs close to CC) or 'canonical' (MICs well above CC) rpoB mutations ${ }^{28,29}$, similar results are also expected for M. tuberculosis isolates with low-level resistance to FQs/SLIDs. The problems associated with slow and/or inaccurate DST of $M$. tuberculosis by phenotypic methods have been overcome by developing molecular methods. Although molecular methods are also not perfect ${ }^{30}$, specific detection of mutations for most anti-TB drugs including FQs and SLIDs correlate well with treatment outcome $\mathrm{e}^{8-10,15,16,28,29}$.

The WHO has recommended the use of line probe assays for rapid first-line and second-line diagnostic screening for MDR-TB and XDR-TB, respectively, as the initial test in place of phenotypic DST ${ }^{1,6}$. This study compared the performance of GenoType MTBDRsl version 1 (gMTBDRslv1) (containing probes targeting gyrA for FQ resistance, rrs for resistance to SLIDs and $e m b B$ codon 306 for EMB resistance) and GenoType MTBDRsl version 2 (gMTBDRslv2) (containing probes targeting $g y r A$ and gyrB for FQ resistance, $r r s$ for resistance to SLIDs and eis for resistance to KAN) tests for the detection of second-line drug resistance among MDR-TB isolates in Kuwait. Results for selected isolates were validated by PCR-sequencing of respective gene loci. Resistance to RIF and INH was also confirmed by using GenoType MTBDRplus or PCR-sequencing tests.

\section{Results}

Clinical specimens and $\boldsymbol{M}$. tuberculosis isolates. The clinical source of $152 \mathrm{M}$. tuberculosis strains used in this study are shown in Table 1 . The MDR-TB strains $(n=102$, representing all available MDR-TB strains collected during 2006 to 2019) were cultured from 78 respiratory and 24 non-respiratory specimens obtained from 102 (males, $\mathrm{n}=54$ ) patients. Simultaneously, 50 pansusceptible $M$. tuberculosis isolates cultured from 34 respiratory and 16 non-respiratory samples collected from 50 patients (males, $n=34$ ) were also used (Table 1). The MDR-TB strains were grown from 14 patients from Gulf Cooperation Council (GCC) countries (including 12 Kuwaiti and 2 Saudi Arabian nationals) and 88 patients from non-GCC countries (expatriate workers or their family dependents comprising Indian, $n=37$; Filipino, $n=17$; Ethiopian, $n=15$; Nepalese, $n=6$; Iraqi, $n=4$; Egyptian, $\mathrm{n}=3$; Bangladeshi, $\mathrm{n}=2$; Indonesian, $\mathrm{n}=2$; Georgian, $\mathrm{n}=1$ and Syrian, $\mathrm{n}=1$ subjects). Pansusceptible strains were grown from Indian $(n=18)$, Filipino $(n=7)$, Kuwaiti $(n=6)$, Bangladeshi $(n=4)$, Nepali $(n=3)$, Sri Lankan $(n=3)$, Ethiopian $(n=2)$, Pakistani $(n=2)$, Syrian $(n=2)$, and 3 other ( 1 each from Indonesia, Sudan and Mali) patients. All isolates were cultured from newly-diagnosed TB patients before initiation of treatment with anti-TB drugs. All MDR-TB $(\mathrm{n}=102)$ and pansusceptible $(\mathrm{n}=50)$ isolates were identified as $M$. tuberculosis complex strains by the AccuProbe DNA probe assay ${ }^{31}$ and by an in-house multiplex PCR assay ${ }^{32}$, as expected. 


\begin{tabular}{|c|c|c|c|c|c|c|c|}
\hline \multirow{2}{*}{\begin{tabular}{|l|} 
Phenotypic resistance \\
of $M$. tuberculosis to \\
\end{tabular}} & \multicolumn{2}{|c|}{$\begin{array}{l}\text { No. of isolates with } r p o B \\
\text { mutation as }\end{array}$} & \multicolumn{5}{|c|}{$\begin{array}{l}\text { No. of isolates detected with a } \\
\text { mutation by gMTBDRsl v1/v2 in }\end{array}$} \\
\hline & S450L & Other mutations $\mathrm{s}^{\mathrm{a}}$ & gyrA & gyrB & rrs & eis & $e m b B$ \\
\hline None $(n=50)$ & 0 & 0 & 0 & 0 & 0 & 0 & 0 \\
\hline $\mathrm{INH}+\mathrm{RIF}(\mathrm{n}=32)$ & 21 & 11 & 2 & 0 & 0 & 0 & 14 \\
\hline $\mathrm{INH}+\mathrm{RIF}+\mathrm{EMB}(\mathrm{n}=5)$ & 2 & 3 & 1 & 0 & 0 & 0 & 4 \\
\hline $\mathrm{INH}+\mathrm{RIF}+\mathrm{STR}(\mathrm{n}=31)$ & 24 & 7 & $6^{\mathrm{b}}$ & 0 & 5 & 0 & 20 \\
\hline $\mathrm{INH}+\mathrm{RIF}+\mathrm{EMB}+\mathrm{STR}(\mathrm{n}=34)$ & 26 & 8 & $3^{\mathrm{c}}$ & 2 & $3^{\mathrm{d}}$ & 1 & 21 \\
\hline Total & 73 & 29 & 12 & 2 & 8 & 1 & 59 \\
\hline
\end{tabular}

Table 2. Phenotypic resistance by MGIT 960 system to SIRE drugs and genotypic screening of mutations in $r p o B$, gyrA, gyrB, rrs and eis among 152 clinical $M$. tuberculosis isolates. INH isoniazid, RIF rifampin, EMB ethambutol, STR streptomycin. ${ }^{\mathrm{a}}$ Other mutations included H445Y/D/R, $\mathrm{n}=12 ; \mathrm{D} 435 \mathrm{~V}, \mathrm{n}=4$; Q432E/K/L/P, $\mathrm{n}=5 ; \mathrm{S} 450 \mathrm{~W}, \mathrm{n}=3$; V170F, $\mathrm{n}=2 ; \mathrm{M} 434 \mathrm{I}+\mathrm{D} 435 \mathrm{Y}, \mathrm{n}=2$ and D435G $+\mathrm{H} 445 \mathrm{Q}, \mathrm{n}=1$. ${ }^{\mathrm{b}}$ Two isolates with $r p o B$ mutation S450W. ${ }^{c}$ One isolate with $r p o B$ mutations $\mathrm{D} 435 \mathrm{G}+\mathrm{H} 445 \mathrm{Q} .{ }^{\mathrm{d}}$ One isolate with $r p o B$ mutation H445R.

Phenotypic DST patterns. The drug-susceptible $M$. tuberculosis isolates $(n=50)$ were susceptible to all 4 SIRE (streptomycin or STR, INH, RIF and EMB) drugs tested (pansusceptible strains). When MDR-TB strains were tested, 32 isolates were resistant to 2 (INH + RIF) drugs, 36 isolates were resistant to 3 (INH $+\mathrm{RIF}+\mathrm{STR}=31$ and $\mathrm{INH}+\mathrm{RIF}+\mathrm{EMB}=5)$ drugs and 34 isolates were resistant to all 4 SIRE drugs (Table 2).

Genotypic detection of resistance to RIF and INH. The GenoType MTBDRplus assay/PCR-sequencing data showed that all pansusceptible strains $(n=50)$ were susceptible to RIF (Table 2) and INH as they yielded wild-type patterns for $r p o B, k a t G$ and $i n h A$. All MDR-TB strains were resistant to RIF based on GenoType MTBDRplus assay/PCR-sequencing data as they exhibited an rpoB mutation. Of 102 MDR-TB strains, 73 and 3 isolates contained S450L and S450W mutation, respectively, while 26 isolates contained mutations at other $r p o B$ codon positions (Table 2). However, GenoType MTBDRplus assay/PCR-sequencing data confirmed the MDRTB status of only 100 isolates as 2 isolates exhibited wild-type pattern for kat $G$ and $i n h A$ suggesting that INH resistance in these 2 isolates either involved mutations in other regions of kat $G$ or $i n h A$ genes or in other genes ${ }^{3}$.

Genotypic detection of resistance to FQs and SLIDs. All 50 pansusceptible $M$. tuberculosis isolates contained wild-type pattern for $g y r A$, rrs and embB codon 306 by gMTBDRslv1 assay and for $g y r A, g y r B, r r s$ and eis genes by gMTBDRslv2 assay (Table 2). When 102 MDR-TB strains were tested by gMTBDRslv1 and gMTBDRslv2 assays, 12 isolates contained a mutation in gyrA (A90V,3; D94G, $\mathrm{n}=5 ; \mathrm{D} 94 \mathrm{~A}, \mathrm{n}=2$ and D94N/Y, $\mathrm{n}=2$ ) while the remaining 90 isolates contained wild-type $g y r A$. Of 2 isolates with D94N/Y mutations in $g y r A$, PCR-sequencing identified D94N and D94Y mutation in 1 isolate each. Of 12 isolates with gyrA mutations, 2 isolates were resistant to INH + RIF only, 7 isolates were resistant to 3 while the remaining 3 isolates were resistant to all 4 SIRE drugs (Table 2). Furthermore, gMTBDRslv1 and gMTBDRslv2 assays detected 8 other isolates with A1401G mutation while the remaining 94 isolates contained wild-type $r r s$. Of 8 isolates with an $r r s$ mutation, 5 isolates were resistant to STR + INH + RIF while the remaining 3 isolates were resistant to all 4 SIRE drugs (Table 2).

The gMTBDRslv1 assay also detected an embB codon 306 mutation in 59 MDR-TB strains (M306V, $\mathrm{n}=32$; M306I, $\mathrm{n}=17$ and 10 isolates with a non-specific mutation detected by lack of hybridization with wild-type probe) while the remaining 43 isolates contained wild-type embB codon 306. Among 10 isolates with a non-specific $e m b B$ mutation, PCR-sequencing of $e m b B$ identified M306I (ATG306ATC) mutation in 6 isolates and M306L (ATG306CTG) mutation in 4 isolates. Of $59 \mathrm{MDR}-\mathrm{TB}$ isolates with embB codon 306 mutations, 14 isolates were resistant to INH + RIF only, 24 isolates were resistant to 3 while the remaining 21 isolates were resistant to all 4 SIRE drugs (Table 2).

The gMTBDRslv2 assay also detected N538D mutation in 1 isolate and a non-specific mutation indicated by lack of hybridization with wild-type (WT) gyrB probe in another isolate. PCR-sequencing of gyrB identified T539N mutation in the latter isolate. The remaining 100 isolates contained wild-type gyrB. Furthermore, 1 isolate contained an eis mutation detected only by lack of hybridization with WT2 eis probe. PCR-sequencing of eis identified G-10A mutation in this isolate. The remaining 101 isolates contained wild-type eis. Both eis and gyrB mutations were detected in isolates resistant to all 4 SIRE drugs (Table 2). The prevalence of mutations conferring resistance to FQs ( $g y r A / g y r B)$ or SLIDs ( rrs/eis) was significantly higher in MDR-TB strains resistant to 3 drugs versus 2 drugs ( 12 of 36 versus 2 of 32, $P=0.007$ ) as well as for all 4 SIRE drugs versus 2 drugs ( 9 of 34 versus 2 of $32, P=0.045)$. Interestingly, the prevalence of mutations conferring resistance to FQs ( $g y r A / g y r B)$ or SLIDs (rrs/ eis) was also higher in MDR-TB strains with an $r p o B$ S450L mutation versus isolates with other $r p o B$ mutations ( 19 of 73 versus 4 of $29, P=0.182$ ), however, the difference was not statistically significant.

The distribution of mutations in $g y r A / g y r B$ or $r r s / e i s$ genes conferring resistance to FQs and SLIDs, respectively, among 23 MDR-TB strains collected over a 14-year period (2006 to 2019) together with mutations in rpoB, $i n h A$ and $e m b B$ genes and fingerprinting data by spoligotyping are presented in Table 3 . No isolate contained a mutation in both $g y r A / g y r B+r r s /$ eis genes, i.e., XDR-TB was not detected in Kuwait (Table 3). The spoligotyping data showed that the majority ( 13 of $21,62 \%$ ) of MDR-TB strains with a mutation in $g y r A / g y r B$ or $r r s /$ eis genes 


\begin{tabular}{|c|c|c|c|c|c|c|c|c|c|c|c|c|c|}
\hline \multirow[b]{2}{*}{ Serial no } & \multirow{2}{*}{$\begin{array}{l}\text { Clinical } \\
\text { specimen }^{\mathrm{a}}\end{array}$} & \multirow[b]{2}{*}{ Isolate no } & \multirow{2}{*}{$\begin{array}{l}\text { Year of } \\
\text { isolation }\end{array}$} & \multirow{2}{*}{$\begin{array}{l}\text { Phenotypic } \\
\text { resistance to }\end{array}$} & \multicolumn{7}{|c|}{ Drug resistance-conferring mutation(s) detected in ${ }^{c}$} & \multicolumn{2}{|c|}{ Spoligotyping data } \\
\hline & & & & & $r p o B$ & inhA & gyrA & gyrB & $r r s$ & eis & $e m b B^{d}$ & SIT $^{\mathrm{e}}$ & Family \\
\hline 1 & Sputum & $8728 / 40$ & 2006 & SIRE & $\mathrm{D} 435 \mathrm{G}+\mathrm{H} 445 \mathrm{Q}$ & WT & D94A & WT & WT & WT & WT & 44 & T5 \\
\hline 2 & Sputum & $3370 / 305$ & 2008 & IRE & S450L & G-17 T & D94G & WT & WT & WT & M306V & 26 & CAS1-Delhi \\
\hline 3 & CSF & $8714 / 770$ & 2009 & SIRE & S450L & WT & WT & T539N & WT & WT & M306V & 244 & Beijing \\
\hline 4 & Sputum & $14,626 / 1189$ & 2009 & SIRE & H445R & WT & WT & WT & A1401G & WT & M306V & 1 & Beijing \\
\hline 5 & Sputum & $7596 / 666$ & 2010 & SIR & S450L & WT & D94A & WT & WT & WT & WT & 49 & $\mathrm{H} 3$ \\
\hline 6 & BAL & $1185 / 768$ & 2011 & IR & S450L & WT & D94G & WT & WT & WT & M306I & 288 & CAS2 \\
\hline 7 & Pus & $3762 / 322$ & 2011 & SIR & S450L & WT & WT & WT & A1401G & WT & WT & 1 & Beijing \\
\hline 8 & LN & \begin{tabular}{|l|}
$1266 / 96$ \\
\end{tabular} & 2013 & SIRE & S450L & WT & WT & N538D & WT & WT & WT & 11 & EAI3-IND \\
\hline 9 & Sputum & $3608 / 216$ & 2013 & SIR & S450L & WT & A90V & WT & WT & WT & M306V & 1 & Beijing \\
\hline 10 & Sputum & \begin{tabular}{|l|}
$463 / 57$ \\
\end{tabular} & 2014 & SIR & S450W & WT & WT & WT & A1401G & WT & WT & 1 & Beijing \\
\hline 11 & BAL & $16,684 / 10$ & 2014 & SIR & S450L & WT & D94N & WT & WT & WT & M306V & 794 & CAS1-Delhi \\
\hline 12 & Sputum & $818 / 153$ & 2015 & SIR & S450L & WT & WT & WT & A1401G & WT & WT & 1 & Beijing \\
\hline 13 & Sputum & $2723 / 225$ & 2015 & SIRE & S450L & WT & D94G & WT & WT & WT & M306V & 1 & Beijing \\
\hline 14 & Sputum & $6633 / 640$ & 2015 & SIRE & S450L & WT & WT & WT & A1401G & WT & WT & 1 & Beijing \\
\hline 15 & Sputum & $10,246 / 708$ & 2015 & SIR & S450L & WT & WT & WT & A1401G & WT & WT & 1 & Beijing \\
\hline 16 & Sputum & $4784 / 255$ & 2016 & SIR & S450W & WT & A90V & WT & WT & WT & M306I & $\mathrm{NA}$ & Orphan \\
\hline 17 & Sputum & $5089 / 283$ & 2016 & SIR & S450L & T-8A & WT & WT & A1401G & WT & M306V & 1 & Beijing \\
\hline 18 & Sputum & $5873 / 294$ & 2016 & SIR & S450L & WT & D94G & WT & WT & WT & WT & 26 & CAS1-Delhi \\
\hline 19 & Sputum & $12,373 / 650$ & 2016 & IR & S450L & WT & D94Y & WT & WT & WT & WT & 1 & Beijing \\
\hline 20 & Sputum & $7730 / 394$ & 2017 & SIRE & S450L & WT & WT & WT & WT & G-10A & WT & 1 & Beijing \\
\hline 21 & Sputum & $16,359 / 764$ & 2017 & SIRE & S450L & WT & WT & WT & A1401G & WT & WT & 1 & Beijing \\
\hline 22 & Sputum & $13,820 / 604$ & 2018 & SIRE & S450L & C-15 T & $\mathrm{A} 90 \mathrm{~V}$ & WT & WT & WT & M306V & ND & NA \\
\hline 23 & CSF & $14,406 / 637$ & 2019 & SIR & S450L & WT & D94G & WT & WT & WT & M306I & ND & NA \\
\hline
\end{tabular}

Table 3. Clinical details and phenotypic and molecular characterization of 23 MDR-TB isolates by GenoType MTBDRsl version 1 and GenoType MTBDRsl version 1 showing genotypic resistance to FQs or SLIDs ${ }^{\mathrm{a}}$. ${ }^{\mathrm{a}} \mathrm{FQs}$, fluoroquinolones, SLIDs, second-line injectable drugs; CSF, cerebrospinal fluid; BAL, bronchoalveolar lavage; LN, lymph node. ${ }^{b}$ S, streptomycin; I, isoniazid; R, rifampin, E, ethambutol. ' All isolates contained S315T mutation in $k a t G$ gene. ${ }^{\mathrm{e}} \mathrm{embB}$ codon 306 mutations only; ${ }^{\mathrm{S} I T}$, shared international type; NA, not available; ND, not done.

belonged to Beijing genotype. All isolates with identical gyrA mutation were genotypically distinct strains based on other mutation patterns and/or by spoligotyping (Table 3). However, 6 of 8 isolates with A1401G mutation in $r r s$ were genetically identical. The year-wise data on the prevalence of $g y r A / g y r B$ mutations conferring resistance to FQs, rrs/eis mutation conferring resistance to SLIDs and $e m b B$ codon 306 mutation conferring resistance to EMB in MDR-TB strains during the 14-year study period are shown in Table 4. When the total 14-year period of the study was split into two 7-year periods, the prevalence of mutations conferring resistance to FQs ( 9 of 47 versus 5 of 55, $P=0.160$ ) or SLIDs ( 7 of 47 versus 2 of 55, $P=0.077$ ) alone was higher in MDR-TB strains collected during 2013 to 2019 compared to 2006 to 2012, however, the differences were not statistically significant (Table 4). On the contrary, the prevalence of mutations conferring resistance to FQs or SLIDs (pre-XDR-TB) was significantly higher during 2013 to 2019 compared to 2006 to 2012 ( 16 of 47 versus 7 of 55, $P=0.016$ ) (Table 4).

\section{Discussion}

Kuwait is an Arabian Gulf country in the Middle East. The total population of 4.8 million individuals in 2019 comprised 30\% Kuwaitis and 70\% expatriate workers or their dependents (https://www.paci.gov.kw/Default. aspx). Most expatriates in Kuwait originate from TB endemic countries of South/Southeast Asia and Africa (such as India, Bangladesh, Pakistan, Philippines, Egypt, Sudan and Ethiopia $)^{31,33,34}$. Kuwait has a low $(\sim 24$ cases per 100000 population) incidence of TB and a low ( 1.1\%) incidence of MDR-TB ${ }^{31,35}$. Nearly $85 \%$ of all TB cases and $>90 \%$ of MDR-TB cases occur in expatriate population ${ }^{31,35}$. These cases mainly arise due to reactivation of latent TB infection acquired previously by TB patients in their respective countries even though all expatriates are screened for the evidence of active TB disease at the time of their entry into Kuwait ${ }^{33-35}$. All clinical specimens from suspected TB patients are tested by GeneXpert MTB/RIF assay for rapid diagnosis of active TB disease and detection of RIF resistance in addition to routine processing for smear microscopy and culture by MGIT 960 system $^{30,31}$. Although phenotypic DST against first-line drugs and STR is performed on all M. tuberculosis isolates for optimal patient management, phenotypic DST against second-line drugs is not performed, mainly due to low rate of MDR-TB ${ }^{31,35}$.

In this study we evaluated the performance of two line probe assays (gMTBDRslv1 and gMTBDRslv2) for rapid detection of mutations conferring resistance to FQs and SLIDs in $M$. tuberculosis and the results were extended by PCR-sequencing of respective gene loci. These assays identified 5 different gyrA mutations at 2 


\begin{tabular}{|l|c|l|l|l|l|l|}
\hline \multirow{2}{*}{ Year } & No. of MDR-TB & \multicolumn{6}{|l|}{$\begin{array}{l}\text { No. of MDR-TB isolates with a } \\
\text { mutation in }\end{array}$} \\
\cline { 2 - 7 } Isolates tested & gyrA & gyrB & rrs & eis & $\boldsymbol{e m b B}^{\text {a }}$ \\
\hline 2006 & 8 & 1 & 0 & 0 & 0 & 2 \\
\hline 2007 & 10 & 0 & 0 & 0 & 0 & 5 \\
\hline 2008 & 7 & 1 & 0 & 0 & 0 & 4 \\
\hline 2009 & 5 & 0 & 1 & 1 & 0 & 4 \\
\hline 2010 & 6 & 1 & 0 & 0 & 0 & 2 \\
\hline 2011 & 10 & 1 & 0 & 1 & 0 & 7 \\
\hline 2012 & 9 & 0 & 0 & 0 & 0 & 5 \\
\hline 2013 & 5 & 1 & 1 & 0 & 0 & 4 \\
\hline 2014 & 8 & 0 & 0 & 1 & 0 & 5 \\
\hline 2015 & 10 & 2 & 0 & 3 & 0 & 6 \\
\hline 2016 & 8 & 3 & 0 & 1 & 0 & 5 \\
\hline 2017 & 7 & 0 & 0 & 1 & 1 & 2 \\
\hline 2018 & 5 & 1 & 0 & 0 & 0 & 4 \\
\hline 2019 & 4 & 1 & 0 & 0 & 0 & 4 \\
\hline Total & 102 & 12 & 2 & 8 & 1 & 59 \\
\hline
\end{tabular}

Table 4. The year-wise prevalence of $g y r A / g y r B, r r s / e i s$ and $e m b B$ mutations in multidrug-resistant $M$. tuberculosis (MDR-TB) isolates analyzed during the 14-year study period. ${ }^{a} e m b B$ codon 306 mutations only.

different codon positions (A90V, 3; D94G, $\mathrm{n}=5$; D94A, $\mathrm{n}=2$; D94N, $\mathrm{n}=1$ and D94Y, $\mathrm{n}=1$ ) in 12 MDR-TB isolates in Kuwait which are also the most frequently observed gyrA mutations from some geographical locations ${ }^{26,27,36-40}$. Furthermore, M. tuberculosis isolates carrying D94G, D94N and D94Y mutations in gyrA exhibit high-level resistance to FQs and cause higher mortality rates among patients infected with such strains ${ }^{9,10}$. Application of gMTBDRslv2 and PCR-sequencing further identified gyrB mutations (N538D and T539N) in 2 additional isolates which have also been described previously among FQ-resistant $M$. tuberculosis isolates ${ }^{36-41}$.

In a systematic review based on 46 studies involving 3,846 unique $M$. tuberculosis isolates with different phenotypic resistance profiles to FQs, Avalos et al. ${ }^{42}$ showed that mutations G88C, S91P, D94G and D94Y in quinolone resistance determining region of $g y r A$ are always associated with FQ-resistant strains. Similarly, N538D and $\mathrm{T} 539 \mathrm{~N}$ in quinolone resistance determining region of $g y r B$ were also always associated with FQ-resistant strains. On the contrary, mutations A90V, D94A and D94N in gyrA were observed in 330 of 1995, 177 of 1995 and 122 of 1995 ofloxacin-resistant isolates but also in 4 of 1572, 1 of 1572 and 1 of 1572 ofloxacin-susceptible isolates, respectively ${ }^{42}$. Several studies have shown that A90V and D94A mutations cause low-level resistance (minimum inhibitory concentrations, MICs of $0.5 \mathrm{mg} / \mathrm{L}$ to $4 \mathrm{mg} / \mathrm{L}$ ) to ofloxacin/levofloxacin while D94G and D94N mutations mostly cause high-level resistance to FQs (MICs of $4 \mathrm{mg} / \mathrm{L}$ to $32 \mathrm{mg} / \mathrm{L})^{27,37-39,43}$. Phenotypic DST of M. tuberculosis for first-line anti-TB drugs; RIF, EMB and PZA is problematic and many isolates with low-level yet clinically significant resistance to RIF and EMB are scored as drug-susceptible strains ${ }^{15-20,28,29,44}$. Similarly, phenotypic DST for FQs is also not perfect as some isolates with low-level resistance and containing specific gyrA (mainly A90V and D94A) mutations yield discordant results ${ }^{25,27,38}$. Taken together, the findings suggest that detection of $g y r A$ mutations in few FQ-susceptible isolates is also very likely due to faulty phenotypic DST data rather than lack of association of gyrA mutations with FQ resistance.

All 23 isolates with $g y r A / g y r B$ or $r r s / e i s$ mutation contained S315T mutation in kat $G$ and 19 of 23 isolates contained S450L mutation in rpoB. The mutation S315T in kat G has minimal effects on fitness of $M$. tuberculosis and isolates with this mutation are more likely to remain virulent, are amenable to further transmission and so acquire resistance to additional drugs ${ }^{3,8,45}$. Similarly, the mutation S450L in $r p o B$ has minimal effects on fitness as $M$. tuberculosis isolates with this mutation usually contain compensatory mutations in $r p o A / r p o C$, remain infectious and thus acquire resistance to additional drugs in new patients ${ }^{3,8,45,46}$. Although the prevalence of mutations conferring resistance to FQs $(g y r A / g y r B)$ and SLIDs ( rrs/eis) in MDR-TB strains was higher in isolates with an $r p o B$ S450L mutation versus isolates with all other mutations (19 of 73 versus 4 of 29), the difference did not reach statistical significance $(P=0.182)$. High prevalence of $g y r A$ and $r r s$ mutations conferring resistance to FQs and SLIDs, respectively, in MDR-TB strains carrying S450L mutation in rpoB gene was also reported from India ${ }^{47}$. The mutation patterns and fingerprinting data by spoligotyping showed that nearly all 14 isolates with a $g y r A / g y r B$ mutation were genotypically distinct strains. These 14 isolates were obtained from expatriate patients with 13 of 14 patients originating from the Indian subcontinent (India, $\mathrm{n}=11$, Bangladesh, $\mathrm{n}=1$; Nepal, $\mathrm{n}=1$ ). The data are consistent with previous observations showing that most MDR-TB strains in Kuwait are genotypically different as they arise mostly as a result of reactivation of latent $M$. tuberculosis infection acquired previously by expatriate patients in their respective countries ${ }^{34,48,49}$. Furthermore, the occurrence of $g y r A$ mutations in MDR-TB strains isolated mostly from Indian patients is consistent with high prevalence of resistance of multidrug-resistant $M$. tuberculosis isolates from India to FQs ${ }^{40,47,50,51}$. Also, the detection of D94G $(n=5)$ and A90V $(n=3)$ mutations in 8 of $10 \mathrm{FQ}$-resistant strains is consistent with the high prevalence of these mutations in MDR-TB strains from India ${ }^{40,47,50,51}$. 
Eight MDR-TB isolates contained A1401G mutation in $r r s$ that confers resistance to SLIDs and 6 of 8 isolates (isolate no. 3762/322, 463/57, 818/153, 6633/640, 10,246/708 and 16,359/764) were genetically similar and were recovered from Kuwaiti patients, likely as a result of local transmission of MDR-TB in Kuwait even though the index case remained elusive ${ }^{34}$. Although phenotypic DST against SLIDs was not performed in this study, all MDR-TB isolates with a defined $r r s$ mutation in a recent study from South Africa showed phenotypic resistance to SLIDs ${ }^{39}$. A multicenter cohort study carried out in seven high TB burden countries has recently shown that inaccurate DST by phenotypic methods leads to under-treatment of drug-resistant TB and increased patient mortality ${ }^{52}$. The study further showed that rapid molecular DST in place of phenotypic DST for first-line and second-line drugs is required for improved outcome for patients with MDR-TB, pre-XDR-TB or XDR-TB ${ }^{52}$.

Although XDR-TB was not detected in Kuwait, the prevalence of mutations conferring resistance to FQs alone ( 9 of 47 versus 5 of 55, $P=0.160$ ) or SLIDs alone ( 7 of 47 versus 2 of $55, P=0.077$ ) was higher, though not statistically significant, in MDR-TB strains collected during 2013 to 2019 compared to 2006 to 2012 . However, the prevalence of mutations conferring resistance to FQs or SLIDs (pre-XDR-TB) was significantly higher during 2013 to 2019 compared to 2006 to 2012 ( 16 of 47 versus 7 of $55, P=0.016$ ) strongly suggesting that the prevalence of these mutations is increasing in Kuwait. The prevalence of resistance to FQs has also increased among MDR-TB strains in recent years in India ${ }^{53-56}$ and China ${ }^{43}$. These findings are relevant as most $(11$ of $14,79 \%)$ MDR-TB strains in Kuwait with gyrA/gyrB mutations were obtained from expatriate patients of Indian origin.

Application of gMTBDRslv1 assay also detected an embB codon 306 mutation in 59 of 102 (58\%) MDR-TB strains. Interestingly, only 25 of these 59 isolates were phenotypically resistant to EMB while the remaining 34 isolates were EMB-susceptible. The embB codon 306 mutations confer low-level resistance to EMB which is often missed by the faulty phenotypic DST methods ${ }^{20-22,24,44}$. Thus rapid detection of EMB resistance in MDRTB strains by gMTBDRslv1 test will avoid treatment with an ineffective drug, evolution of further resistance and drug-related toxicity ${ }^{57}$.

Our study has a few limitations. (1) Phenotypic DST of pansusceptible and MDR-TB strains against FQs and SLIDs was not performed, (2) The presence of mutations in gyrA/gyrB genes conferring resistance to FQs and in rrs/eis genes conferring resistance to SLIDs by gMTBDRslv1 and gMTBDRslv12 tests was carried out in culture isolates and not directly on clinical samples obtained from TB patients and (3) The outcome of infection among MDR-TB patients was not available as most patients were expatriates who were sent back to their respective countries after the initial objective of sputum smear-negative status was achieved.

In conclusion, our results show that combined use of gMTBDRslv1 and gMTBDRslv2 assays in 102 MDRTB strains detected mutations in 12 and 2 isolates in $g y r A$ and $g y r B$, respectively conferring resistance to FQs and in 8 and 1 isolate in $r r s$ and eis, respectively, conferring resistance to SLIDs. Mutations in $e m b B$ were also detected in 59 MDR-TB isolates. These mutations were absent among 50 pansusceptible M. tuberculosis isolates. Although XDR-TB was not detected, an increasing trend in the frequency of pre-XDR-TB was evident in Kuwait as the frequency of mutations conferring resistance to FQs or SLIDs was significantly higher among isolates collected during 2013-2019 versus 2006-2012. Application of both tests is warranted for proper management of MDR-TB patients in Kuwait, a low TB/MDR-TB setting, as gMTBDRslv2 detected resistance to FQs and/or SLIDs in 3 additional isolates while gMTBDRslv1 additionally detected resistance to EMB in $58 \%$ of MDR-TB isolates. The $e m b B$ mutations confer low-level resistance to EMB which is often missed by the faulty phenotypic DST methods and their detection in MDR-TB strains by gMTBDRslv1 will avoid treatment with an ineffective drug and drug-related toxicity.

\section{Materials and methods}

Clinical specimens and $\boldsymbol{M}$. tuberculosis isolates. A total of 102 MDR-TB isolates cultured during 2006 to 2019 from 102 TB patients (representing all available MDR-TB strains) and 50 randomly selected $M$. tuberculosis isolates fully susceptible to first-line anti-TB drugs (pansusceptible strains) collected from 50 TB patients were analyzed. The MDR-TB strains were grown from 78 respiratory and 24 non-respiratory specimens while pansusceptible strains were cultured from 34 respiratory and 16 non-respiratory clinical specimens. All clinical specimens were obtained from suspected TB patients after obtaining verbal consent only as part of routine patient care, diagnostic work-up and resistance surveillance at Kuwait National TB Control Laboratory (KNTCL) before initiation of treatment with anti-TB drugs. The study was approved by the Health Sciences Center Ethical Committee, Kuwait University (Approval no. VDR/EC/3451 dated 18-12-2018) and all experimental procedures and investigations were performed in accordance with their guidelines and regulations. Since the study did not involve direct contact with patients and the results are reported on deidentified samples without revealing patient identity, the need for informed consent was waived by the Health Sciences Center Ethical Committee.

Sterile clinical specimens were processed directly for the cultivation of mycobacteria whereas non-sterile samples were processed by using $N$-acetyl-L-cysteine and sodium hydroxide (NALC/NaOH) at KNTCL for culture. The cultures were grown in liquid media-based automated mycobacteria growth indictor tube (MGIT) 960 system according to manufacturer's instructions (Becton Dickinson, Sparks, MD, USA) and as described previously ${ }^{31,44}$. All MGIT cultures were positive for the presence of acid-fast bacilli (AFB) by Ziehl-Neelsen smear microscopy and for the presence of M. tuberculosis complex DNA by AccuProbe DNA probe assay, performed as described previously ${ }^{31}$.

Phenotypic DST by Bactec MGIT 960 system. Phenotypic DST of all M. tuberculosis isolates was performed with the automated MGIT 960 system for RIF, INH, EMB and STR by using the SIRE drug kit used according to manufacturer's instructions and as described previously ${ }^{31,44}$. The KNTCL regularly participates in drug susceptibility proficiency testing. 
Genotypic characterization. Genotypic characterization was performed at Reference Mycobacteriology Laboratory, Department of Microbiology, Faculty of Medicine, Kuwait University. For this purpose, genomic DNA was obtained from MGIT 960 system cultures by using Chelex-100 as described previously ${ }^{58}$. All MGIT 960 system cultures were first tested for the presence of $M$. tuberculosis complex DNA by an in-house multiplex PCR assay, performed as described previously ${ }^{32}$, and for mutations in genes conferring resistance to first-line (RIF and INH) and second-line (FQs and SLIDs) anti-TB drugs.

Detection of mutations conferring resistance to RIF and INH. All isolates were tested by commercial GenoType MTBDRplus line probe assay (Hain Lifesciences, Nehren, Germany) for the detection of mutations in hot-spot region of $r p o B$ gene for RIF resistance and for detection of mutations at kat $G$ codon 315 $(k a t G)$ and $i n h A$ regulatory region (inhA) for INH resistance, according to kit instructions and as described previously ${ }^{59}$. Water was used in place of DNA for negative controls. PCR-sequencing of hot-spot region and $\mathrm{N}$-terminal region of $r p o B$ gene and $k a t G$ and $i n h A$ was carried out for isolates showing no mutation or a nonspecific mutation indicated by lack of hybridization with a wild-type probe only. DNA sequencing was performed as described previously ${ }^{59}$. M. tuberculosis codon numbering system was used for the $r p o B$ gene ${ }^{60}$.

\section{Detection of mutations conferring resistance to FQs and SLIDs}

The gMTBDRslv1 contains probes targeting gyrA for FQ resistance, $r r s$ for resistance to SLIDs and embB gene codon 306 for EMB resistance. The gMTBDRslv2 contains probes targeting gyrA and gyrB for FQ resistance, $r r s$ for resistance to SLIDs and $e i s$ for resistance to $\mathrm{KAN}^{36}$. Both gMTBDRslv1 and gMTBDRslv2 were obtained commercially (Hain Lifesciences) and were used according to kit instructions. Lack of detection of specific mutation or detection of resistance only by lack of hybridization with wild-type probes was confirmed by PCR amplification followed by DNA sequencing (PCR-sequencing) of the corresponding gene fragment.

The gyrA gene was amplified by using GYRAF (5'-CGCAGCTACATCGACTATGCGATG-3') and GYRAR (5'-GGGATGAAATCGATGTCTCCTCG-3') primers and PCR amplification protocol described previously ${ }^{58}$, the amplicons were purified by using PCR product purification kit (Qiagen, Hilden, Germany) used according to kit instructions and both strands were sequenced by using internal sequencing primer GYRAFS ( $5^{\prime}$-CGG GTGCTCTATGCAATGTTC-3') or GYRARS (5'-GGCTTCGGTGTACCTCATCGCC-3') by using the DNA sequencing protocol described previously ${ }^{34,59}$. The gyrB gene was amplified by using GYRBF (5'-CAAGTCCGA ACTGTATGTCGTA-3') and GYRBR (5'-CCGCATGAACCGGAACAACAA-3') primers, the amplicons were purified and both strands were sequenced as described above except that sequencing primer GYRBFS ( $5^{\prime}$-TCC GAACTGTATGTCGTAGAAG-3') or GYRBRS (5'-ATGAACCGGAACAACAACGTCA-3') was used.

The $3^{\prime}$-end of $r$ s (16S rRNA) gene was amplified by using 16S3F (5'-GCGATGCCGCGAGGTTAAGCGAA$3^{\prime}$ ) and 16S3R (5'-CCAACAGTGTGTTGGTGGCCAA-3') primers, the amplicons were purified and both strands were sequenced as described above except that 16S3FS (5'-ATCCTTAAAAGCCGGTCTCAGT-3') or 16S3RS (5'-CTCCTTAGAAAGGAGGTGATCCA-3') was used as sequencing primer. The $5^{\prime}$-end of eis gene was amplified by using EIS5F (5'-CCAGCGTAACGTCACGGCGAA-3') and EIS5R (5'-GCACCGTCAACCGCAGAT CCA-3') primers, the amplicons were purified and both strands were sequenced as described above except that EIS5FS (5'-CACGGCGAAATTCGTCGCTGA-3') or EIS5RS (5'-GCAGATCCATGTACAGCGCCA-3') was used as sequencing primer. The $\mathrm{embB}$ codon 306 region was amplified by using EMB306A (5'-CCGACGCCGTGG TGATATTCGGCT-3') and EMB306B (5'-GTAATACCAGCCGAAGGGATCCTC-3') primers and the purified amplicons were sequenced as described previously ${ }^{20}$. Nucleotide and amino acid sequences of the amplified products were compared with wild-type sequence from $M$. tuberculosis $\mathrm{H}_{37} \mathrm{Rv}$ using Clustal omega.

Spoligotyping. Fingerprinting of M. tuberculosis isolates carrying mutations in target genes conferring resistance to FQs and SLID was carried out by spoligotyping, performed as described previously ${ }^{34}$. The spoligotyping results in binary format were used for the assignment of different phylogenetic lineages according to SITVIT database (http://www.pasteur-guadeloupe.fr:8081/SITVITDemo/index.jsp). The spoligotyping patterns not described previously in SITVIT2 database were designated as 'orphan' patterns ${ }^{34}$.

Statistical analyses. Statistical analyses were performed by using Fisher's exact (two-tailed) test or Pearson's chi-square test as appropriate and probability levels $<0.05$ were considered as significant. Statistical analyses were performed by using WinPepi software ver. 11.65 (PEPI for Windows, Microsoft Inc., Redmond, WA, USA).

Received: 3 December 2020; Accepted: 30 March 2021

Published online: 08 April 2021

\section{References}

1. World Health Organization. Global Tuberculosis Report 2020. https://www.who.int/tb/publications/global_report/en/ (WHO, 2020).

2. World Health Organization. Drug-Resistant TB, Surveillance and Response: Supplement to Global Tuberculosis Report 2014 (WHO/ $\mathrm{HQ} / \mathrm{TB} / 2014.12$, WHO, 2014).

3. Ahmad, S. \& Mokaddas, E. Current status and future trends in the diagnosis and treatment of drug-susceptible and multidrugresistant tuberculosis. J. Infect. Pub. Health 7, 75-91 (2014).

4. Dheda, K. et al. The epidemiology, pathogenesis, transmission, diagnosis, and management of multidrug-resistant, extensively drug-resistant, and incurable tuberculosis. Lancet Resp. Med. 5, 291-360 (2017).

5. World Health Organization. WHO Treatment Guidelines for Rifampicin- and Multidrug-Resistant Tuberculosis. https://www.who. int/tb/areas-of-work/drug-resistant-tb/guideline-update2018/en/ (2018).

6. World Health Organization. Global Tuberculosis Report 2019 (WHO/CDS//TB/2019.15. WHO, 2019). 
7. Horne, D. J. et al. Diagnostic accuracy and reproducibility of WHO-endorsed phenotypic drug susceptibility testing methods for first-line and second-line antituberculosis drugs. J. Clin. Microbiol. 51, 393-401 (2013).

8. Miotto, P., Zhang, Y., Cirillo, D. M. \& Yam, W. C. Drug resistance mechanisms and drug susceptibility testing for tuberculosis. Respirology 23, 1098-1113 (2018).

9. Rigouts, L. et al. Specific gyrA gene mutations predict poor treatment outcome in MDR-TB. J. Antimicrob. Chemother. 71, 314-323 (2016).

10. Georghiou, S. B., Seifert, M., Catanzaro, D. G., Garfein, R. S. \& Rodwell, T. C. Increased tuberculosis patient mortality associated with Mycobacterium tuberculosis mutations conferring resistance to second-line antituberculous drugs. J. Clin. Microbiol. 55, 1928-1937 (2017).

11. Sarathy, J. et al. Fluoroquinolone efficacy against tuberculosis is driven by penetration into lesions and activity against resident bacterial populations. Antimicrob. Agents Chemother. 63, e02516-e2518 (2019).

12. Ahmad, S. \& Mokaddas, E. Recent advances in proper management of multidrug-resistant tuberculosis. Kuwait Med. J. 50, 146-160 (2018).

13. Kendall, E. A., Cohen, T., Mitnick, C. D. \& Dowdy, D. W. Second line drug susceptibility testing to inform the treatment of rifampinresistant tuberculosis: a quantitative perspective. Int. J. Infect. Dis. 56, 185-189 (2017).

14. Walzl, G. et al. Tuberculosis: advances and challenges in development of new diagnostics and biomarkers. Lancet Infect. Dis. 18, e199-e210 (2018).

15. Rigouts, L. et al. Rifampin resistance missed in automated liquid culture system for Mycobacterium tuberculosis isolates with specific rpoB mutations. J. Clin. Microbiol. 51, 2641-2645 (2013).

16. Van Deun, A. et al. Rifampin drug resistance tests for tuberculosis: challenging the gold standard. J. Clin. Microbiol. 51, 2633-26340 (2013).

17. Al-Mutairi, N. M., Ahmad, S., Mokaddas, E., Eldeen, H. S. \& Joseph, S. Occurrence of disputed rpoB mutations among Mycobacterium tuberculosis isolates phenotypically susceptible to rifampicin in a country with a low incidence of multidrug-resistant tuberculosis. BMC Infect. Dis. 19, 3 (2019).

18. Mvelase, N. R. et al. rpoB mutations causing discordant rifampicin susceptibility in Mycobacterium tuberculosis: retrospective analysis of prevalence, phenotypic, genotypic, and treatment outcomes. Open Forum Infect. Dis. 6, ofz065 (2019).

19. Torrea, G. et al. Variable ability of rapid tests to detect Mycobacterium tuberculosis rpoB mutations conferring phenotypically occult rifampicin resistance. Sci. Rep. 9,11826 (2019).

20. Ahmad, S., Jaber, A. A. \& Mokaddas, E. Frequency of $e m b B$ codon 306 mutations in ethambutol-susceptible and -resistant clinical Mycobacterium tuberculosis isolates in Kuwait. Tuberculosis (Edinburgh) 87, 123-129 (2007).

21. Plinke, C., Walter, K., Aly, S., Ehlers, S. \& Niemann, S. Mycobacterium tuberculosis embB codon 306 mutations confer moderately increased resistance to ethambutol in vitro and in vivo. Antimicrob. Agents Chemother. 55, 2891-2896 (2011).

22. Cheng, S., Cui, Z., Li, Y. \& Hu, Z. Diagnostic accuracy of a molecular drug susceptibility testing method for the antituberculosis drug ethambutol: a systematic review and meta-analysis. J. Clin. Microbiol. 52, 2913-2924 (2014).

23. Cambau, E. et al. Revisiting susceptibility testing in MDR-TB by a standardized quantitative phenotypic assessment in a European multicentre study. J. Antimicrob. Chemother. 70, 686-696 (2015).

24. Al-Mutairi, N. M., Ahmad, S. \& Mokaddas, E. Molecular screening versus phenotypic susceptibility testing of multidrug-resistant Mycobacterium tuberculosis isolates for streptomycin and ethambutol. Microb. Drug Resist. 24, 923-931 (2018).

25. Coeck, N. et al. Correlation of different phenotypic drug susceptibility testing methods for four fluoroquinolones in Mycobacterium tuberculosis. J. Antimicrob. Chemother. 71, 1233-1240 (2016).

26. Maningi, N. E., Malinga, L. A., Antiabong, J. F., Lekalakala, R. M. \& Mbelle, N. M. Comparison of line probe assay to BACTEC MGIT 960 system for susceptibility testing of first and second-line anti-tuberculosis drugs in a referral laboratory in South Africa. BMC Infect. Dis. 17, 795 (2017).

27. Huo, F. et al. Specific gyrA gene mutations correlate with high prevalence of discordant levofloxacin resistance in Mycobacterium tuberculosis isolates from Beijing, China. J. Mol. Diagn. 22, 1199-1204 (2020).

28. Ho, J., Jelfs, P. \& Sintchencko, V. Phenotypically occult multidrug-resistant Mycobacterium tuberculosis: dilemmas in diagnosis and treatment. J. Antimicrob. Chemother. 68, 2915-2920 (2013).

29. Shah, N. S. et al. Clinical impact on tuberculosis treatment outcomes of discordance between molecular and growth-based assays for rifampin resistance, California 2003-2013. Open Forum Infect. Dis. 3, ofw150 (2016).

30. Mokaddas, E., Ahmad, S., Eldeen, H. S. \& Al-Mutairi, N. Discordance between Xpert MTB/RIF assay and Bactec MGIT 960 culture system for detection of rifampin-resistant Mycobacterium tuberculosis isolates in a country with a low tuberculosis (TB) incidence. J. Clin. Microbiol. 53, 1351-1354 (2015).

31. Mokaddas, E., Ahmad, S. \& Samir, I. Secular trends in susceptibility patterns of Mycobacterium tuberculosis isolates in Kuwait, 1996-2005. Int. J. Tuberc. Lung Dis. 12, 319-325 (2008).

32. Mokaddas, E. \& Ahmad, S. Development and evaluation of a multiplex PCR for rapid detection and differentiation of Mycobacterium tuberculosis complex members from non-tuberculous mycobacteria. Jpn. J. Infect. Dis. 60, 140-144 (2007).

33. Ahmad, S., Al-Mutairi, N. M. \& Mokaddas, E. Variations in the occurrence of specific rpoB mutations in rifampicin-resistant Mycobacterium tuberculosis strains isolated from patients of different ethnic groups in Kuwait. Indian J. Med. Res. 135, 756-762 (2012).

34. Al-Mutairi, N. M., Ahmad, S. \& Mokaddas, E. Molecular characterization of multidrug-resistant Mycobacterium tuberculosis (MDR-TB) isolates identifies local transmission of infection in Kuwait, a country with a low incidence of TB and MDR-TB. Eur. J. Med. Res. 24, 38 (2019).

35. Ahmad, S., Mokaddas, E. \& Al-Mutairi, N. M. Epidemiology of tuberculosis and multidrug-resistant tuberculosis in the Middle East Region. Expert Rev. Anti Infect. Ther. 16, 709-721 (2018).

36. Brossier, F. et al. Performance of the new version (v2.0) of the GenoType MTBDRsl test for detection of resistance to second-line drugs in multidrug-resistant Mycobacterium tuberculosis complex strains. J. Clin. Microbiol. 54, 1573-1580 (2016).

37. Cui, Z., Wang, J., Lu, J., Huang, X. \& Hu, Z. Association of mutation patterns in $g y r A / B$ genes and ofloxacin resistance levels in Mycobacterium tuberculosis isolates from East China in 2009. BMC Infect. Dis. 11, 78 (2011).

38. Malik, S., Willby, M., Sikes, D., Tsodikov, O. V. \& Posey, J. E. New insights into fluoroquinolone resistance in Mycobacterium tuberculosis: functional genetic analysis of gyrA and gyrB mutations. PLoS ONE 7, e39754 (2012).

39. Gardee, Y. et al. Evaluation of the GenoType MTBDRsl version 2.0 assay for second-line drug resistance detection of Mycobacterium tuberculosis isolates in South Africa. J. Clin. Microbiol. 55, 791-800 (2017).

40. Rufai, S. B., Umay, K., Singh, P. K. \& Singh, S. Performance of genotype MTBDRsl V2.0 over the genotype MTBDRsl V1 for detection of second line drug resistance: an Indian perspective. PLoS ONE 15, e0229419 (2020).

41. Pantel, A. et al. Extending the definition of the GyrB quinolone resistance-determining region in Mycobacterium tuberculosis DNA gyrase for assessing fluoroquinolone resistance in M. tuberculosis. Antimicrob. Agents Chemother. 56, 1990-1996 (2012).

42. Avalos, E. et al. Frequency and geographic distribution of $g y r A$ and $g y r B$ mutations associated with fluoroquinolone resistance in clinical Mycobacterium tuberculosis isolates: a systematic review. PLOS ONE 10, e0120470 (2015).

43. Huo, F. et al. Increased prevalence of levofloxacin-resistant Mycobacterium tuberculosis in China is associated with specific mutations within the gyrA gene. Int. J. Infect. Dis. 92, 241-246 (2020). 
44. Ahmad, S., Mokaddas, E., Al-Mutairi, N., Eldeen, H. S. \& Mohammadi, S. Discordance across phenotypic and molecular methods for drug susceptibility testing of drug-resistant Mycobacterium tuberculosis isolates in a low TB incidence country. PLoS ONE 11, e0153563 (2016).

45. Borrell, S. \& Gagneux, S. Infectiousness, reproductive fitness and evolution of drug-resistant Mycobacterium tuberculosis. Int. J. Tuberc. Lung Dis. 13, 1456-1466 (2009).

46. Song, T. et al. Fitness costs of rifampicin resistance in Mycobacterium tuberculosis are amplified under conditions of nutrient starvation and compensated by mutation in the $\beta$ ' subunit of RNA polymerase. Mol. Microbiol. 91, 1106-1119 (2014).

47. Ajbani, K., Nikam, C., Shetty, A., Soman, R. \& Rodrigues, C. Multidrug-resistant tuberculosis with fluoroquinolone resistance: sinister association with other drugs and ominous implications for treatment. Clin. Infect. Dis. 59, 138-139 (2014).

48. Ahmad, S., Mokaddas, E. \& Fares, E. Characterization of rpoB mutations in rifampin-resistant Mycobacterium tuberculosis isolates from Kuwait and Dubai. Diagn. Microbiol. Infect. Dis. 44, 245-252 (2002).

49. Ahmad, S. \& Mokaddas, E. The occurrence of rare rpoB mutations in rifampicin-resistant Mycobacterium tuberculosis isolates from Kuwait. Int. J. Antimicrob. Agents 26, 205-212 (2005).

50. Ajbani, K. et al. Evaluation of genotype MTBDRsl assay to detect drug resistance associated with fluoroquinolones, aminoglycosides and ethambutol on clinical sediments. PLOS ONE 7, e49433 (2012).

51. Chandak, R. J. et al. Evaluation of MTBDRsl for detecting resistance in Mycobacterium tuberculosis to second-line drugs. Int. J. Tuberc. Lung Dis. 23, 1257-1262 (2019).

52. Zürcher, K. et al. Drug susceptibility testing and mortality in patients treated for tuberculosis in high-burden countries: a multicentre cohort study. Lancet Infect. Dis. 19, 298-307 (2019).

53. Dalal, A. et al. Resistance patterns among multidrug-resistant tuberculosis patients in greater metropolitan Mumbai: trends over time. PLoS ONE 10, e0116798 (2015).

54. Goyal, V., Kadam, V., Narang, P. \& Singh, V. Prevalence of drug-resistant pulmonary tuberculosis in India: systematic review and meta-analysis. BMC Public Health 17, 817 (2017).

55. Sharma, N. et al. Pattern and trends of drug sensitivity in MDR-TB cases in Delhi (2009-2014): a record based study. Indian J. Tuberc. 66, 222-226 (2019).

56. Singh, P. K., Singh, U. \& Jain, A. Emergence of Specific gyrA mutations associated high-level fluoroquinolone-resistant Mycobacterium tuberculosis among multidrug-resistant tuberculosis cases in North India. Microb. Drug Resist. 2020 Sep 29. https://doi. org/10.1089/mdr.2020.0240. Online ahead of print.

57. Jin, K. W., Lee, J. Y., Rhiu, S. \& Choi, D. G. Longitudinal evaluation of visual function and structure for detection of subclinical ethambutol-induced optic neuropathy. PLoS ONE 14, e0215297 (2019).

58. Ahmad, S., Fares, E., Araj, G. F., Chugh, T. D. \& Mustafa, A. S. Prevalence of S315T mutation within the kat G gene in isoniazidresistant clinical Mycobacterium tuberculosis isolates from Dubai and Beirut. Int. J. Tuberc. Lung Dis. 6, 920-926 (2002).

59. Al-Mutairi, N., Ahmad, S. \& Mokaddas, E. Performance comparison of four methods for rapid detection of multidrug-resistant Mycobacterium tuberculosis strains. Int. J. Tuberc. Lung Dis. 15, 110-115 (2011).

60. Andre, E. et al. Consensus numbering system for the rifampicin resistance-associated rpoB gene mutations in pathogenic mycobacteria. Clin. Microbiol. Infect. 23, 167-172 (2017).

\section{Acknowledgements}

This study was supported by Kuwait University Research Sector Grant No. MI01/18.

\section{Author contributions}

Conceived and designed the experiments: N.A.M., S.A., and E.M. Performed the experiments: N.A.M. Analyzed the data: N.A.M., S.A., and E.M. Contributed reagents/materials/analysis tools: S.A., and E.M. Wrote the paper: N.A.M., S.A., and E.M.

\section{Competing interests}

The authors declare no competing interests.

\section{Additional information \\ Correspondence and requests for materials should be addressed to S.A.}

Reprints and permissions information is available at www.nature.com/reprints.

Publisher's note Springer Nature remains neutral with regard to jurisdictional claims in published maps and institutional affiliations.

(c) (i) Open Access This article is licensed under a Creative Commons Attribution 4.0 International

License, which permits use, sharing, adaptation, distribution and reproduction in any medium or format, as long as you give appropriate credit to the original author(s) and the source, provide a link to the Creative Commons licence, and indicate if changes were made. The images or other third party material in this article are included in the article's Creative Commons licence, unless indicated otherwise in a credit line to the material. If material is not included in the article's Creative Commons licence and your intended use is not permitted by statutory regulation or exceeds the permitted use, you will need to obtain permission directly from the copyright holder. To view a copy of this licence, visit http://creativecommons.org/licenses/by/4.0/.

(C) The Author(s) 2021 